
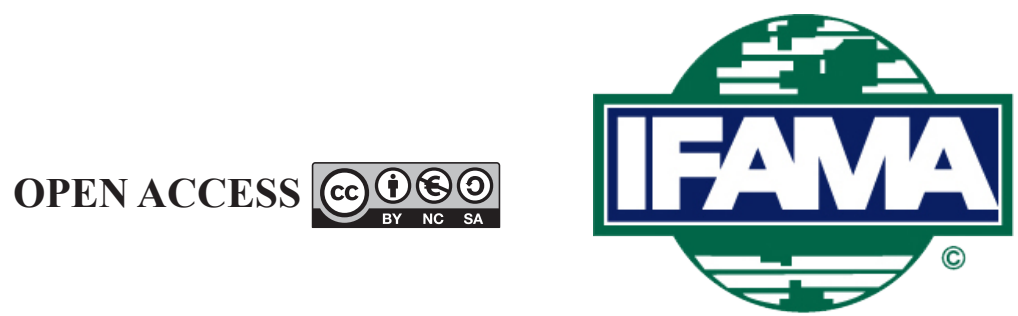

International Food and Agribusiness Management Review

Volume 24, Issue 5, 2021; DOI: 10.22434/IFAMR2020.0162

Received: 15 September 2020 / Accepted: 30 December 2020

\title{
Understanding the low use rate of food nutrition information in China
}

\section{RESEARCH ARTICLE}

\author{
Lijun Guan $^{\mathrm{a}}$, Yan Zhang ${ }^{\mathrm{b}}$, Shaosheng Jin ${ }^{\circledR \mathrm{c}}$ and Lin Zhou ${ }^{\mathrm{d}}$ \\ a Assistant Professor, School of Economics, Zhejiang University of Technology, \\ 288 Liuhe Road, Hangzhou 310023, China P.R.
}

${ }^{b}$ Assistant Professor, State Key Laboratory of Grassland Agro-ecosystems; College of Pastoral Agriculture Science and Technology, Lanzhou University, 222 Tianshui Road, Lanzhou 730000, China P.R.

'Professor, China Academy for Rural Development (CARD), School of Public Affairs, Zhejiang University, 866 Yuhangtang Road, Hangzhou 310058, China P.R.

${ }^{d}$ Researcher, Institute of Food and Nutrition Development, Ministry of Agriculture, No. 12 Zhongguancun South St., Haidian District, Beijing 100081, China P.R.

\begin{abstract}
Based on a survey of 1,225 consumers in 10 cities of five representative provinces, this study was designed to understand the low use rate of food nutrition information in China after a mandatory label policy was instituted in 2013. We found that consumers always neglect nutrition information at point-of-purchase. But they refer to simplified front-of-pack labels more frequently than detailed back-of-pack labels. Fat information is most concerned especially by females; whereas sodium information attracts the least concern. Ignorance, a belief that the information is un-useful are the main cause of the low use rate of nutritional information. Thus, simplified and striking tag formats should be used to capture consumers' attention and convince them of the importance of nutrition information. The findings can also be used as a guide in the design of food marketing programs directing at different consumer groups for food processors targeting the Chinese market.
\end{abstract}

Keywords: nutritional information, label scheme, ignorance, attitudes, China JEL code: I12, Q13, Q18

(1)Corresponding author: ssjin@zju.edu.cn 


\section{Introduction}

The consumption of processed foods is increasing rapidly in low-income and middle-income countries because they have enormous commercial advantages, including durability, palatability, immediate edibility (Popkin, 2014), and lower relative prices (Machado et al., 2017). In China, the consumption of processed foods is increasing at a rate of 50\% each year (Zhou et al., 2015). In 2011, 28.5\% of the total daily energy intake of Chinese adults came from processed foods, and this proportion was as high as $40.2 \%$ for children and teenagers in megacities (Zhou et al., 2015). The growth of processed food has become one of the major reasons for the prevalence of overweight and obesity (Bonanno and Goetz, 2012; Louzada et al., 2015) because processed foods are always associated with higher levels of saturated fats, sodium, added sugar, and energy, but are typically low in dietary fiber and micronutrients (Moubarac et al., 2013). According to the Chinese Residents Nutrition and Chronic Disease Status Report (2015), the overweight rate in adults aged over 18 years throughout the country is $30.15 \%$ and the obesity rate is $11.9 \%$, showing increases of 7.3 and $4.8 \%$, respectively, since 2002. Considering absolute numbers, China has 43.2 million obese men and 46.4 million obese women becoming the country with the highest number of obese people worldwide due to the size of its population (NCD Risk Factor Collaboration, 2016).

Providing nutritional information on the package of foods is one of the most important measures taken by governments around the world to constrain obesity rates. Firstly, nutrition labeling is an essential instrument for reducing asymmetric and uncertain information by providing them with information on nutrient contents (Akerlof, 1970; Roe et al., 2014). It increases consumers' confidence in the food market (Houghton et al., 2008), and helps consumers especially those who are looking for special products to compare various foods (Lin et al., 2014; Miller and Cassady, 2012). Secondly, nutrition labeling also influences the general perception of the health qualities of foods and reduces the attractiveness of unhealthy food (Boztug et al., 2015). It can help consumers choose a healthier diet by reducing the intake of calories and fat (Shimokawa, 2016), and increasing the intake of fiber and vitamin (Grasso et al., 2017).

In 2013, the Chinese government made it mandatory for food processors to disclose nutritional information on prepackaged foods by implementing the National Food Safety Standard for Prepackaged Food Labeling (GB 7718-2011). This aims to help consumers choose healthier foods by giving them access to consistent, easy-to-read, and trustworthy nutritional information. Under this regulation, Nutrition Facts Panels on the back of the package must display the amount of energy, protein, fats, carbohydrates, and sodium contained in a product in a standardized format, with their percentages relative to the nutrient reference value. And nutrition claims on the front of the package can only be displayed on the products which meet the specified criteria.

It is imperative that research be undertaken to understand the use of food nutritional information after this policy was instituted. However, few studies have focused on emerging countries such as China. Research conducted before the label policy by Liu et al. (2015) reported a low frequency of Nutrition Facts Panel label usage $(28.5 \%)$ by Chinese consumers. In contrast, there has been considerable research into nutritional labeling in the USA and European countries (e.g. Grunert and Wills, 2007; Wang et al., 2016). Generally, $40-60 \%$ of consumers in American and European countries are reported to use nutritional labels frequently, and most consumers are capable of understanding nutritional labels well.

Based on a nationwide sample of 1,225 Chinese consumers, this study was designed to understand the low use rate of food nutritional information in China after the mandatory label policy. To ensure a deep analysis, both detailed Nutrition Facts Panels and simplified Nutritional Claim labels, as well as five kinds of nutrients information including energy, protein, fat, carbohydrate, and sodium were considered. Two research questions were specifically addressed: (1) How often do Chinese consumers use different kinds of food nutritional information after the implementation of the mandatory labeling policy in 2013? (2) What factors explain the low use rate of nutritional information in China? For the remainder of the paper, we will first summarize the background of the food labeling policy in China. Then, the national survey data together with the analytical model are specified. This is followed by the results and discussion, and finally conclusion and implications. 


\section{Background of the food labeling policy in China}

China's food labeling policy can be divided into three parts (Table 1). Firstly, food label policy provides general guidance on what should be labeled applying to all prepackaged food. China's first food label policy (GB 7718-1987) was published in 1987, then revised separately in 1994 and 2004. However, these regulations were all voluntary for general food enterprises, failing to get important consideration neither by food industries nor consumers. In 2011, the third amendment of the general food label standard (GB 77182011) modified the labeling methods of food additives, the specifications for the labeling method, and the contents of allergy-causing substances, making it first mandatory for all food enterprises.

Secondly, special dietary label policy standardizes the labeling of the energy and nutrient contents of special foods, including infant foods and nutrient-intensified foods, to meet the requirements of infants, ill individuals, and other special groups. China released the first special dietary label policy in 1992 (GB 13432-1992), then revised it and made it mandatory in 2004 (GB 13432-2004). It required the producers of prepackaged special dietary foods to include the contents of protein, fat, carbohydrate, and other nutrients and regulated the mode of presentation of and the allowable deviations from these energy and nutrients labeling requirements. Although only applied in special dietary products, GB 13432-2004 is the first mandatory labeling policy in China laying an important foundation for the follow-up mandatory policies.

Thirdly, nutrition label policy refers to the labeling related to the description of and facts about nutrient contents. Chinese first nutrition label policy was not formalized until 2007. It stated that energy, protein, fat, carbohydrate, and sodium contents should be described first in the Nutrition Facts Panels, together with any nutritional or functional claims. This regulation also specified a set of requirements for the label form, location, font, sequence, and so on. Then, in 2011, the Chinese first mandatory nutrition label policy was developed (GB 28050-2011), which came into effect in January 2013. Under this regulation, no prepackaged food can be sold unless it supplies the required nutrient content information. Furthermore, the precisely stipulated values corresponding to the nutrient contents must meet any nutritional claims, such as high calcium, low fat, and so on. This labeling standard marked a new stage in the standardization and regulation of label management in China. For the first time, it incorporated both nutrition and health in the overall food safety strategy.

Table 1. Development of food labeling policy in China.

\begin{tabular}{lc}
\hline $\begin{array}{l}\text { Classification } \\
\text { Title of law or regulation (number or publication date) }\end{array}$ & $\begin{array}{c}\text { Voluntary/ } \\
\text { Mandatory }\end{array}$ \\
\hline Food label & Voluntary \\
\hline General standard for the labeling of foods (GB 7718-1987) & Voluntary \\
General standard for the labeling of foods (GB 7718-1994) & Voluntary \\
General standard for the labeling of prepackaged foods (GB 7718-2004) & Mandatory \\
\hline National food safety standard for prepackaged food labeling (GB 7718-2011) & \\
\hline Special dietary label & Voluntary \\
\hline Labeling of foods for special nutrients (GB 13432-1992) & Mandatory \\
General standard for the labeling of prepackaged foods for special dietary uses (GB 13432-2004) & Mandatory \\
National food safety standard for prepackaged food labeling for special dietary uses & \\
\hline (GB 13432-2013) & Voluntary \\
\hline Nutrition label & Mandatory \\
\hline Food nutrition labeling management regulation (2007) & National food safety standard for prepackaged food nutrition labeling (GB 28050-2011) \\
\hline
\end{tabular}




\section{Methods}

\subsection{National survey data}

Data used in this study were obtained with nationwide face-to-face interviews undertaken in 2016. We used a multistage, random cluster process to randomly draw the sample surveyed from each province and city. We selected five provinces, Hebei, Guangdong, Zhejiang, Sichuan, and Hubei Province, representing the north, south, east, west, and middle of China, respectively. In each province, we randomly chose a developed and an underdeveloped city, according to the per capita gross domestic product in 2013 (China Statistical Yearbook, 2014). After a pretest was completed in Hangzhou, Zhejiang Province, the formal investigation was conducted from mid-July to mid-August 2016. The sample surveyed included individuals entering supermarkets or stores randomly chosen in each city. The participants all received ten yuan for their patience and time. Finally, a 1,225 Chinese resident sample was obtained, and the response rate is $96.46 \%$.

The survey was found to have good validity and reliability. We measured the internal consistency separately for each section. Cronbach's $\alpha$ for each section was more than 0.7, greater than the minimum requirement for internal consistency recommended by Kline (1993). And the sample was roughly consistent with the China Statistics Yearbook (2015) in terms of age, marital status, and household size (Table 2). The respondents were aged $18-82$ years, with an average age of 34.66 years. Of all the respondents, $60.66 \%$ were married, and the average household size was 3.57 family members. Males comprised $46.45 \%$ of the sample, which was slightly lower than the census data; but because women are the main shoppers in Chinese households, our sample is reasonable.

\subsection{Empirical model}

We assumed that a consumer's net utility from nutritional labels depends upon several attributes of the consumers and the food products. The net utility of label use is a function of vector $X$, which is composed of six attributes: demographic characteristics $\left(\mathrm{x}_{1}\right)$, habit-related variables $\left(\mathrm{x}_{2}\right)$, attitude to labeling $\left(\mathrm{x}_{3}\right)$, dietary habits $\left(\mathrm{x}_{4}\right)$, health status $\left(\mathrm{x}_{5}\right)$, and product factors $\left(\mathrm{x}_{6}\right)$. The empirical model is given by:

$$
y^{*}=\beta^{\prime} X_{i}+\varepsilon
$$

Where $y^{*}$ is the net utility of either: (a) back-of-pack Nutrition Facts Panel label; or (b) front-of-pack Nutrition Claim label; or (c) specific nutrient content in the Nutrition Facts Panel, including energy, protein, fat, carbohydrate, or sodium (Figure 1). As mentioned previously, $X$ is a vector of the control variables, which include six distinct categories, defined in detail in Table 2. $\beta$ is the parameter column vector; and $\varepsilon$ is the random error term that allows for uncertainty.

Net utility $y^{*}$ is a latent variable which we cannot observe directly. And $\mathrm{Y}_{i}$ is a random variable that indicates the observed frequency of nutritional label use by consumer i. For each type of label or nutrient, the respondents were asked: 'In general, how often do you look for this information on a food package while shopping', with a five-point answering scale: $1=$ always, $2=$ often, $3=$ sometimes, $4=$ seldom, and $5=$

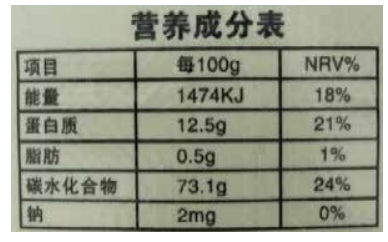

A

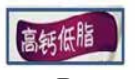

B

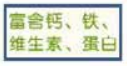

$\mathrm{C}$

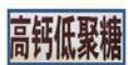

$\mathrm{D}$

Figure 1. Nutrition Facts Panel (A) and Nutrition Claims (B, C, D) in China. 
Table 2. Statistical analysis and definitions of independent variables.

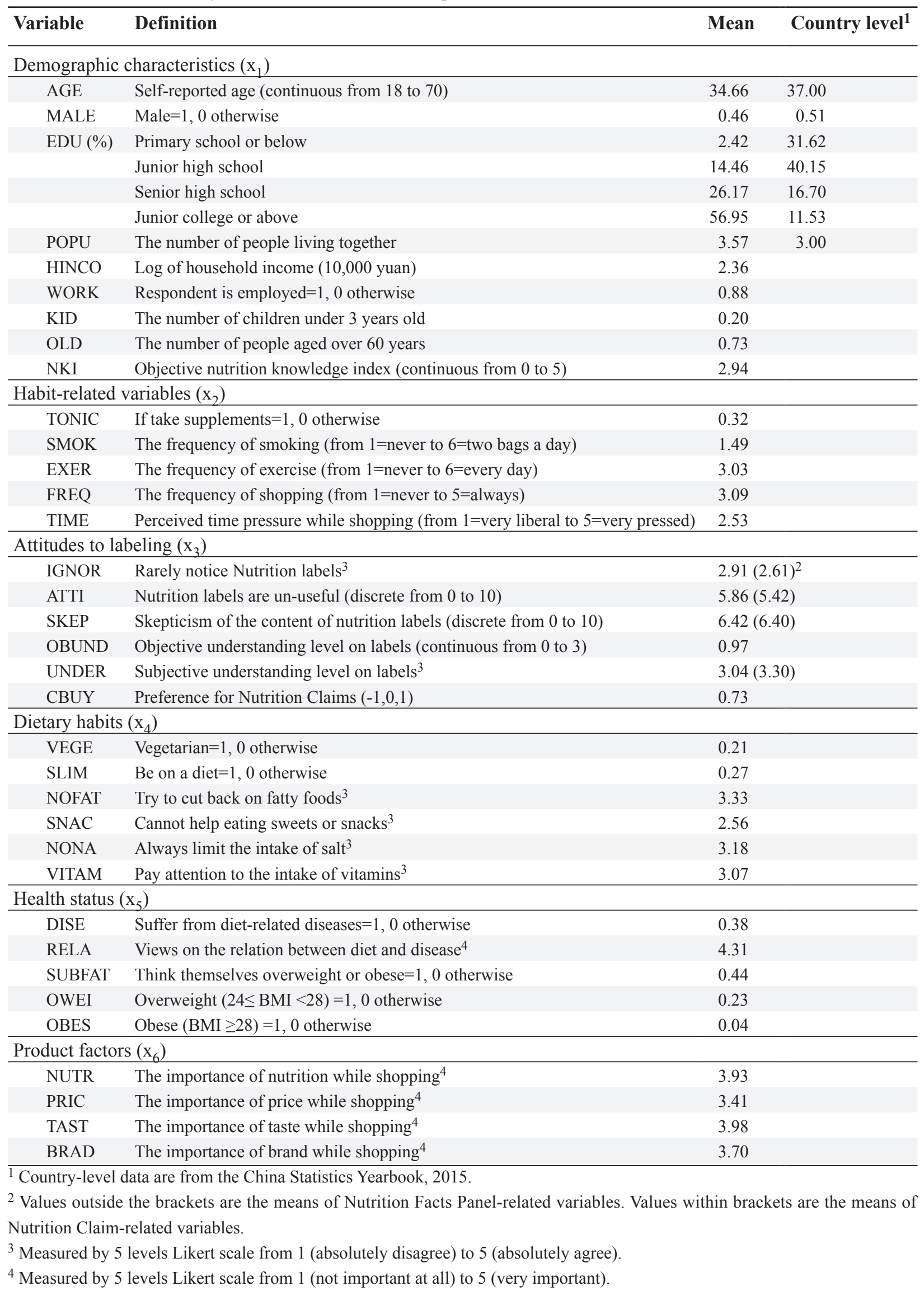


never. Traditionally, $\beta$ can be estimated from the ordered logit model because the frequencies are ranked in order (Long, 1997). However, an important assumption for ordinal logistic regression models is the parallel lines assumption, that the parameters do not change for different categories. As shown in Table 3, five kinds of tests were used to test the parallel lines assumption (Long, 1997). Because the assumption did not hold in all of the tests (all $P$-values $<0.01$ ), we estimated partial proportional odds models (PPOM), as suggested by Peterson and Harrell (1990), instead of the ordered logit model (Williams, 2006).

A key advantage of PPOM is that it allows some of the coefficients in $\beta$ to be the same for all values of $\mathrm{j}$ in the following equation, while others can differ. If we assume that the random error term $\varepsilon$ follows a logistic distribution, with a mean of 0 and a variance of $\pi^{2} / 3$, then the cumulative probability equation for PPOM can be written as:

$$
\mathrm{P}\left(\mathrm{Y}_{\mathrm{i}} \leq j\right)=\frac{\exp \left(\mu_{j}-\beta_{j}^{\prime} X_{i}\right)}{1+\left[\exp \left(\mu_{j}-\beta_{j}^{\prime} X_{i}\right)\right]}=\Lambda\left(\mu_{j}-\beta_{j}^{\prime} X_{i}\right) \quad, j=1,2,3,4
$$

$j=1,2,3,4$, represents the changes in consumers' nutrition label usage frequency, from never to seldom, from seldom to sometimes, from sometimes to often, from often to always respectively. The probabilities of $Y_{i}$ can be expressed as:

$$
\begin{aligned}
& \mathrm{P}\left(\mathrm{Y}_{\mathrm{i}}=1\right)=\Lambda\left(\mu_{1}-\beta_{1}{ }^{\prime} X_{i}\right) \\
& \mathrm{P}\left(\mathrm{Y}_{\mathrm{i}}=2\right)=\Lambda\left(\mu_{2}-\beta_{2}{ }^{\prime} X_{i}\right)-\Lambda\left(\mu_{1}-\beta_{1}{ }^{\prime} X_{i}\right) \\
& \mathrm{P}\left(\mathrm{Y}_{\mathrm{i}}=3\right)=\Lambda\left(\mu_{3}-\beta_{3}{ }^{\prime} X_{i}\right)-\Lambda\left(\mu_{2}-\beta_{2}{ }^{\prime} X_{i}\right) \\
& \mathrm{P}\left(\mathrm{Y}_{\mathrm{i}}=4\right)=\Lambda\left(\mu_{4}-\beta_{4}{ }^{\prime} X_{i}\right)-\Lambda\left(\mu_{3}-\beta_{3}{ }^{\prime} X_{i}\right) \\
& \mathrm{P}\left(\mathrm{Y}_{\mathrm{i}}=5\right)=1-\Lambda\left(\mu_{4}-\beta_{4}{ }^{\prime} X_{i}\right)
\end{aligned}
$$

The log-likelihood function can be written as:

$$
\begin{aligned}
\mathrm{LnL}=\sum_{i=1}^{n}\{ & P\left(\mathrm{Y}_{\mathrm{i}}=1\right) \ln \Lambda\left(\mu_{1}-\beta_{1}{ }^{\prime} X_{i}\right)+P\left(\mathrm{Y}_{\mathrm{i}}=2\right) \ln \left[\Lambda\left(\mu_{2}-\beta_{2}{ }^{\prime} X_{i}\right)-\Lambda\left(\mu_{1}-\beta_{1}{ }^{\prime} X_{i}\right)\right]+ \\
& P\left(\mathrm{Y}_{\mathrm{i}}=3\right) \ln \left[\Lambda\left(\mu_{3}-\beta_{3}{ }^{\prime} X_{i}\right)-\Lambda\left(\mu_{2}-\beta_{2} X_{i}\right)\right]+ \\
& \left.P\left(\mathrm{Y}_{\mathrm{i}}=4\right) \ln \left[\Lambda\left(\mu_{4}-\beta_{4}{ }^{\prime} X_{i}\right)-\Lambda\left(\mu_{3}-\beta_{3} X_{i}\right)\right]+P\left(\mathrm{Y}_{\mathrm{i}}=5\right) \ln \left[1-\Lambda\left(\mu_{4}-\beta_{4}{ }^{\prime} X_{i}\right)\right]\right\}
\end{aligned}
$$

The unknown parameters $\mu_{1}, \mu_{2}, \mu_{3}, \mu_{4}$, and the parameter vector $\beta$ can be estimated simultaneously with the maximum likelihood method.

\subsection{Endogeneity tests}

Some variables in this study such as objective nutrition knowledge may be endogenous. Over time, a consumer reads more and more nutrition labels, and this allows him/her to compare the various nutrients between different foods. Therefore, nutrition knowledge may increase with more-frequent label use. Thus, we tested

Table 3. Tests of the parallel regression assumption.

\begin{tabular}{llll}
\hline Methods & $\mathbf{C h i}^{\mathbf{2}}$ & df & P>Chi' $^{\mathbf{2}}$ \\
\hline Wolfe Gould & 142.8 & 102 & 0.005 \\
Brant & 139.4 & 102 & 0.008 \\
Score & 155.9 & 102 & 0.000 \\
Likelihood ratio & 157.7 & 102 & 0.000 \\
Wald & 162.2 & 102 & 0.000 \\
\hline
\end{tabular}


for the endogeneity of objective nutrition knowledge using Hausman's specification test (Hausman, 1978) and the Durban-Wu-Hausman (DWH) test (Davidson and MacKinnon, 1993). The results of all endogeneity tests did not reject the null hypothesis, indicating that endogeneity was not an important problem in this study (Table 4).

The results may be explained by the following two aspects of current China. First, Chinese consumers' usage rate of nutrition labels is much lower than that in developed countries; and they understand and remember the nutrition information on the labels poorly (Liu et al., 2015). Therefore, it is difficult for Chinese consumers to acquire nutrition knowledge or change diet habits according to label information. Second, no mandatory Chinese labeling policy was instituted until January 2013. Besides, there has been no national or regional education campaign to explain the meanings of nutrition labels or to promote nutrition labeling until now which contributes little to consumers' individual characteristics such as nutrition knowledge. In conclusion, endogeneity did not severely affect this sample, and Chinese consumers, therefore, constitute a useful population in which to directly assess the effects of various variables including nutrition knowledge on label use.

\section{Findings and discussion}

\subsection{Use of two kinds of nutrition labels}

For two kinds of nutrition labels, the respondents paid more attention to simplified Nutrition Claims than detailed Nutrition Facts Panels. Specifically, only 17.45 and $4.52 \%$ of respondents stated that they often or always used Nutrition Facts Panels when shopping. The situation in China is in sharp contrast to those in America and Europe, where 40-60\% of adults frequently used nutritional labels (e.g. Boztuğ et al., 2015; Sun et al., 2015;). In another Asian country, Korea, more than 34\% of shoppers were frequent users (Kim et al., 2016). Our results are even lower than those in previous studies conducted in China before the mandatory labeling policy was instituted (e.g. Liu et al., 2015), which showed that $28.7 \%$ of consumers often or always use Nutrition Facts Panels. This discrepancy may have arisen because we used a nationwide sample in our study, whereas the data in the previous studies were collected in only one or two cities in China. For simplified Nutrition Claims, about $41.20 \%$ of respondents often or always use Nutrition Claims, twice the proportion who frequently used Nutrition Facts Panels. This was not simply because the Nutrition Claims are much easier to understand, but also because they are more strongly highlighted on the fronts of the packages than the Nutrition Facts Panels on the backs of the packages. Front-of-package label with simplified information or displayed using colors and pictures makes nutrition information more available and increases consumers' attention (Aschemann-Witzel et al., 2013). It can also help consumers better understand the label content and choose a healthier diet (Khandpur et al., 2017).

\subsection{Use of five nutrients in Nutrition Facts Panels}

Of the five different nutrient contents presented on the Nutrition Facts Panels, the consumers were most concerned about fat, where $35.78 \%$ of consumers reported frequently use fat information. This is possibly because fat is a kind of negative information. It has been reported by Burton et al. (1999) that negative information, such as fat content, attracts more attention than positive information, such as vitamin content. This finding indicates that consumers are more motivated to avoid the undesirable consequences of negative nutrients than to enjoy the benefits of positive nutrients. However, sodium, another nutrient presented as

Table 4. Endogeneity tests for nutrition knowledge.

\begin{tabular}{lll}
\hline Models & Hausman test: $\boldsymbol{\chi}^{\mathbf{2}}$ & Durban-Wu-Hausman test: $\boldsymbol{\chi}^{\mathbf{2}}$ \\
\hline Nutrition Knowledge Index - Nutrition Facts Panel & $0.42(P=0.52)$ & $0.44(P=0.51)$ \\
Nutrition Knowledge Index - Nutrition Claim & $1.39(P=0.24)$ & $1.47(P=0.22)$ \\
\hline
\end{tabular}


negative information, attracted the least concern of Chinese consumers. Only $15.99 \%$ of consumers reported that they often or always used sodium information. According to Wang et al. (2011), the Chinese consume over $12 \mathrm{~g}$ of sodium per person per day, twice the maximum intake recommended by the World Health Organization. But Chinese consumers rarely attend to sodium information on the nutrition labels. This is possibly because the sodium information occurs at the bottom of the Nutrition Facts Panel, where it is easily ignored.

\subsection{Factors affecting the use of Nutrition Facts Panels and Nutrition Claims}

Table 5 summarizes the results of the partial proportional odds regression models, using the frequencies of Nutrition Facts Panel use and Nutrition Claims use as the dependent variables. The pseudo- $\mathrm{R}^{2}$ values for both equations were about 0.20 , indicating that the estimated equations respectably fit the survey data.

Of the demographic factors examined, EDU (the level of education) played a positive role to increase consumers' use frequency of Nutrition Facts Panels from often to always, but has no significant impact at low-frequency use levels. Possible explanations for this result are that respondents with higher education level may be more concerned about health and nutrition, and also more literate, allowing them to comprehend Nutrition Facts Panels, which are more complex than Nutrition Claims (Lin and Yen, 2008; Moore et al., 2018). Interestingly, HINCO (household income) was negatively associated with the use of Nutrition Facts Panels, demonstrating that respondents from the higher-income household are less likely to use Nutrition Facts Panels. This is possibly because the higher household income group can buy more-expensive foods and is always confident of the nutritional and health status of these foods (Drichoutis, 2005). Unfortunately, NKI (objective nutrition knowledge) had no significant effect on Nutrition Facts Panel use. However, the significant positive effects of NKI on the use of Nutrition Claims showed that nutrition knowledge can help to promote the use rate of simplified Nutrition Claims. Although Nutrition Facts Panels are more complex and require more knowledge to understand than Nutrition Claims, it is placed on the back of the package and rarely attended to in the consumers' decision process (Peschel et al., 2019). Our results confirm that Chinese consumers with greater knowledge of nutrition are more likely to use simplified front-of-pack labels, but not back-of-back labels with detailed information.

Of the habit-related factors, respondents who shopped more frequently (FREQ) tended to use both kinds of nutrition labels more frequently possibly because they are more familiar with nutritional labels. And familiarity is a key factor in information processing and label use (Grunert and Wills, 2007). The estimates of EXER (the frequency of exercise) and TONIC (the use of health supplements) were consistent and positive but were only statistically significant for Nutrition Facts Panels. These results indicate that respondents who participated in physical exercise or took supplements used Nutrition Facts Panels more frequently, partly because they were more health-conscious (Rimal et al., 2000). Although related research (e.g. Drichoutis, 2005) has reported that respondents with greater time pressure are less likely to read nutritional labels in developed countries, we found no statistically significant effect of TIME indicating that time pressure is not an important factor on label use in China.

Attitudes to labeling were identified as important causes of the low use of nutritional labels in China. The negative estimates for IGNOR (rarely notice labels) and ATTI (the belief that these labels are un-useful) indicated that the respondents who were unaware of nutritional labels on the food packages or who regarded the labels as useless were less likely to use either Nutrition Facts Panels or Nutrition Claims. Chinese consumers consider nutritional labels virtually useless because they find labels too hard to interpret or do not know how to use nutritional information to make a decision. However, SKEP (skeptical attitudes towards the accuracy of the label content) did not play a significant role in their use. This is inconsistent with the findings of Sun et al. (2015), who reported that consumers' trust in and ethical evaluation of the honesty of statements made by companies affected their search for nutritional information. This may be possibly because most Chinese consumers have confidence in the scientific basis of nutritional labels in this survey. UNDER (subjective understanding) was significantly positive in increasing the use frequency of Nutrition Facts Panels from 
Table 5. Marginal effects for the use of nutrition facts panels and nutrition claims. ${ }^{1}$

\begin{tabular}{|c|c|c|c|c|c|c|c|c|}
\hline \multirow[t]{2}{*}{ Variable } & \multicolumn{4}{|c|}{ Nutrition facts panels } & \multicolumn{4}{|c|}{ Nutrition claims } \\
\hline & $1^{2}$ & 2 & 3 & 4 & 1 & 2 & 3 & 4 \\
\hline AGE & -0.01 & -0.01 & -0.01 & -0.01 & 0.01 & 0.01 & 0.01 & 0.01 \\
\hline MALE & -0.45 & 0.09 & -0.25 & 0.10 & -0.25 & -0.25 & -0.25 & -0.25 \\
\hline EDU & 1.31 & 0.28 & 0.14 & $0.81^{* * *}$ & 0.32 & 0.32 & 0.32 & 0.32 \\
\hline POPU & 0.05 & 0.05 & 0.05 & 0.05 & -0.04 & -0.04 & -0.04 & -0.04 \\
\hline HINCO & $-0.18^{*}$ & $-0.18^{*}$ & $-0.18^{*}$ & $-0.18^{*}$ & -0.02 & -0.02 & -0.02 & -0.02 \\
\hline WORK & -0.70 & 0.40 & $-1.13^{* *}$ & -0.26 & 0.37 & 0.37 & 0.37 & 0.37 \\
\hline KID & -0.21 & -0.21 & -0.21 & -0.21 & 0.37 & $-0.38^{*}$ & -0.16 & 0.34 \\
\hline OLD & 0.15 & -0.06 & 0.06 & $0.27^{* *}$ & -0.01 & -0.01 & -0.01 & -0.01 \\
\hline NKI & 0.09 & 0.09 & 0.09 & 0.09 & $0.35^{* * *}$ & $0.35^{* * *}$ & $0.35^{* * *}$ & $0.35^{* * *}$ \\
\hline TONIC & $0.25^{*}$ & $0.25^{*}$ & $0.25^{*}$ & $0.25^{*}$ & 0.17 & 0.17 & 0.17 & 0.17 \\
\hline SMOK & 0.10 & 0.10 & 0.10 & 0.10 & 0.07 & 0.12 & 0.00 & -0.16 \\
\hline EXER & $0.40^{* *}$ & $0.40^{* *}$ & $0.40^{* *}$ & $0.40^{* *}$ & 0.21 & 0.21 & 0.21 & 0.21 \\
\hline FREQ & $0.30^{* * *}$ & $0.30^{* * *}$ & $0.30^{* * *}$ & $0.30^{* * *}$ & $0.28^{* * *}$ & $0.28^{* * *}$ & $0.28^{* * *}$ & $0.28^{* * *}$ \\
\hline TIME & -0.05 & -0.05 & -0.05 & -0.05 & -0.02 & -0.02 & -0.02 & -0.02 \\
\hline CBUY & - & - & - & - & -0.20 & $0.33^{*}$ & $0.62^{* * *}$ & $1.27^{* * *}$ \\
\hline IGNOR & $-0.42^{* * *}$ & $-0.42^{* * *}$ & $-0.42^{* * *}$ & $-0.42^{* * *}$ & $-0.38^{* *}$ & $-0.66^{* * *}$ & $-0.57^{* * *}$ & $-0.31^{* *}$ \\
\hline ATTI & $-0.21^{* * *}$ & $-0.21^{* * *}$ & $-0.21^{* * *}$ & $-0.21^{* * *}$ & -0.02 & $-0.14^{* * *}$ & $-0.13^{* *}$ & $-0.28^{* * *}$ \\
\hline SKEP & 0.00 & 0.00 & 0.00 & 0.00 & 0.00 & 0.00 & 0.00 & 0.00 \\
\hline OBUND & 0.01 & 0.01 & 0.01 & 0.01 & $0.31^{*}$ & 0.07 & -0.13 & 0.14 \\
\hline UNDER & $0.29^{*}$ & $0.18^{*}$ & 0.01 & 0.13 & -0.03 & -0.03 & -0.03 & -0.03 \\
\hline VEGE & 0.14 & 0.14 & 0.14 & 0.14 & 0.15 & $0.50^{* *}$ & 0.34 & -0.41 \\
\hline SLIM & $-0.46^{* *}$ & $-0.46^{* *}$ & $-0.46^{* *}$ & $-0.46^{* *}$ & 0.25 & -0.11 & 0.04 & $-0.86^{* *}$ \\
\hline NOFAT & 0.08 & 0.08 & 0.08 & 0.08 & $0.33^{* *}$ & 0.01 & 0.03 & 0.07 \\
\hline SNAC & 0.00 & 0.00 & 0.00 & 0.00 & 0.06 & 0.06 & 0.06 & 0.06 \\
\hline NONA & $0.32^{*}$ & $0.21^{* *}$ & $0.12^{*}$ & -0.06 & 0.06 & 0.06 & 0.06 & 0.06 \\
\hline VITAM & 0.10 & 0.10 & 0.10 & 0.10 & $0.44^{* * *}$ & $0.22^{* * *}$ & 0.03 & 0.08 \\
\hline DISE & 0.08 & 0.08 & 0.08 & 0.08 & 0.06 & 0.06 & 0.06 & 0.06 \\
\hline RELA & 0.20 & 0.23 & -0.04 & $0.28^{*}$ & 0.12 & 0.12 & 0.12 & 0.12 \\
\hline SUBFAT & 0.26 & 0.26 & 0.26 & 0.26 & -0.02 & -0.02 & -0.02 & -0.02 \\
\hline OWEI & 0.01 & 0.01 & 0.01 & 0.01 & 0.13 & 0.13 & 0.13 & 0.13 \\
\hline OBES & $-0.77^{*}$ & $-0.77^{*}$ & $-0.77^{*}$ & $-0.77^{*}$ & -0.51 & -0.51 & -0.51 & -0.51 \\
\hline NUTR & $2.48^{* *}$ & 0.84 & 0.45 & -0.35 & 0.49 & 0.49 & 0.49 & 0.49 \\
\hline PRIC & -0.38 & -0.38 & -0.38 & -0.38 & 0.14 & 0.14 & 0.14 & 0.14 \\
\hline TAST & -0.09 & -0.09 & -0.09 & -0.09 & -0.08 & -0.08 & -0.08 & -0.08 \\
\hline BRAD & 0.77 & -0.47 & -0.20 & $-0.71^{* *}$ & 0.07 & 0.07 & 0.07 & 0.07 \\
\hline Region & Yes & & & & Yes & & & \\
\hline cons & $9.44^{* * *}$ & $4.87^{* * *}$ & -1.47 & -1.39 & $9.56^{* * *}$ & $3.36^{* * *}$ & 1.02 & -0.36 \\
\hline$\overline{\text { Pseudo }^{2}}$ & 0.1951 & & & & 0.2055 & & & \\
\hline$\chi^{2}$ & 696.67 & & & & 718.13 & & & \\
\hline$P>\chi^{2}$ & 0.0000 & & & & 0.0000 & & & \\
\hline $\mathrm{N}$ & 1,225 & & & & 1,225 & & & \\
\hline
\end{tabular}

$21,2,3,4$, represents the changes in consumers' nutrition label usage frequency, from never to seldom, from seldom to sometimes, from sometimes to often, from often to always respectively. 
never to seldom and from seldom to sometimes. However, OBUND (objective understanding) did not affect Nutrition Facts Panel use. These results indicate that if low-frequency users consider themselves capable of comprehending nutritional labels, they were more likely to read Nutrition Facts Panels, whereas those who can understand the labels did not witness greater interest in referring to Nutrition Facts Panels for more information. Our results are supported by Liu et al. (2015) and expose a profound dissonance in China between the ability to comprehend nutritional information and seeking such information. A possible explanation is that many Chinese consumers regard reading nutritional labels as un-useful or time-consuming. As shown in this survey, $33.06 \%$ of the consumers reported that reading nutritional labels is un-useful; whereas $44.73 \%$ of the consumers regarded nutrition labels as a useful tool.

In terms of dietary habits, the respondents who always limited their intake of salt (NONA) were more likely to read Nutrition Facts Panels. Unfortunately, people who were struggling to lose weight (SLIM) used Nutrition Facts Panels less frequently than other respondents, indicating that nutritional information is typically ignored or underutilized by dieters. In contrast, consumers in Europe or America are more likely to use nutrition information if they keep on a special diet (Kim et al., 2000; Unnevehr and Jagmanaite, 2008). Respondents who paid special attention to their intake of vitamins (VITAM) were more inclined to read Nutrition Claims from never to seldom and from seldom to sometimes, possibly because information about vitamins regularly appears in claim labels.

Among the health status factors, only OBES (obesity) correlated significantly but negatively with the use of Nutrition Facts Panels. This indicates that respondents who were obese in our sample were less motivated to use Nutrition Facts Panels than normal-weight respondents. In comparison, overweight or obese people in Europe and America tend to use nutrition labels more frequently (Blitstein and Evans, 2006; Drichoutis et al., 2008). A possible explanation for underutilizing nutrition labels by obese Chinese people is that they attach more importance to taste, and less importance to nutrition.

Product attribute factors had little effect in our study. The NUTR (nutritional characteristics of a product) had a positive effect on the use of Nutrition Facts Panels from never to seldom, indicating that those who never used Nutrition Facts Panels but attached importance to nutrition tend to increase use frequency of Nutrition Facts Panels from never to seldom. The BRAD (importance of brand) had a negative sign on the use of Nutrition Facts Panels from often to always, implying that frequent users who value products brand as important are less likely to use Nutrition Facts Panel, which is consistent with many previous studies (e.g. Barreiro-Hurlé et al., 2010; Verbeke, 2006). This is possibly because a brand is an indicator of quality for many brand loyalists. Therefore, they only choose their trusted brands, rather than consulting label information.

\subsection{Factors affecting the use of nutrient information}

This paper also summarizes the results of the partial proportional odds regression models in which the use frequencies of information on the five nutrients on the Nutrition Facts Panels are the dependent variables (Supplementary Tables S1-S3). The pseudo- $\mathrm{R}^{2}$ values for the five models were between 0.11 and 0.15 , which implies that the estimated equations are reliable.

IGNOR and ATTI had statistically significant negative coefficients for all five nutrients. Again, our results identified ignorance of labeling and an attitude that this information was un-useful as the most important factors explaining the low use of various types of nutritional information in China. FREQ (shopping frequency) was positively associated with the use of information on energy, protein, fat, and carbohydrate, demonstrating that Chinese consumers who shopped more often used the information on these nutrients more frequently, although this was not true for Sodium. This may be attributable to the fact that information on sodium was widely neglected by Chinese consumers, no matter how often they shopped for foods.

Demographic factors played a more significant role in the energy information usage. Although insignificant effects were found in the Nutrition Facts Panel model, the coefficients for AGE (self-reported age) were 
significantly negative in the energy equation, indicating that elderly people were less likely to read energy information. This is possibly because elderly people attend nutrition facts panel labels but they have difficulty in understanding the nutrient content information in it. Thus, the use frequency of nutrition facts panel label between consumers of different ages is similar, but elderly people got less energy information from it. However, respondents with jobs (WORK) were more attentive to energy information when they read Nutrition Facts Panel labels, possibly because they are more energy-consuming than non-workers. Unlike the Nutrition Facts Panel model, NKI, SMOK (frequency of smoking), OBUND (objective understanding of nutrition labels), and VITAM were all positively significant in the use of the energy information. These results show that nutritional knowledge and understanding ability can help to read energy information. Respondents who smoked habitually were more likely to read energy information than nonsmokers, which is inconsistent with previous studies (e.g. Drichoutis et al., 2009). A possible explanation is that smokers in China are aware of the dangers of smoking but do not want to quit. Therefore, other health-inducing measures, such as attention to nutrients content information, are taken to compensate for the risk conferred by smoking.

Dietary habits and product factors were identified as important explanations of protein information use. The significantly positive estimates for VEGE (vegetarians) and NONA (those who restrict their salt intake) indicate that vegetarians and those who limit their intake of salt are more inclined than others to read protein labels. This is possibly because the diet of vegetarians, which contains no meat, lacks sufficient protein, so these people may be more health-conscious than nonvegetarians. The significant coefficient of TAST implies a negative relationship between taste and the use of protein labels, indicating that consumers who rate taste as important are less interested in the protein content of foods.

In terms of the use of fat information, MALE was negatively significant, indicating that males are less interested in reading fat labels than females. The positive estimate of NOFAT (the preference for reducedfat foods) variable showed that respondents who avoided fat paid special attention to fat labels. The sign of RELA (perception of the relationship between diet and disease) was positive, indicating that people who attach importance to the relationship between diet and disease were more likely to read fat labels. This may be partly explained by the fact that fat is a kind of negative information among the five nutrients cited on labels and is closely related to overweight and obesity, as well as to many chronic diseases.

Concerning the carbohydrate and sodium equations, the sign of TONIC was significantly positive, indicating that the custom of taking supplements promotes the use of both nutrients. Respondents with larger households (POPU) were more likely to use sodium labels. This is possibly because when families are large, the likelihood that members have special dietary habits or diseases related to excessive salt intake increases. Furthermore, in multi-person households, the benefits of label use extend to more people, and the relative value of the time spent in obtaining information on sodium increases.

\section{Conclusions and implications}

The consumption of processed foods is growing rapidly all over the world and is widely considered a major cause of the prevalence of overweight and obesity. Nutritional labels, such as back-of-pack Nutrition Facts Panel and front-of-pack Nutrition Claims, are always considered the best available tool to help consumers choose healthier diets. Since 2013, the Chinese Government has made it mandatory for food processors to disclose the nutritional characteristics of prepackaged foods. Using a nationwide sample of 1,225 individuals, this study was designed to understand the situation and causes of the low use rate of nutritional information after the mandatary labeling policy in China.

Firstly, this study displayed the low use rate of food nutrition information in China, but consumers prefer simplified front-of-pack nutrition claims more than detailed back-of-pack nutrition facts panels. When assessed the use of information on the five main nutrients, we found that the most frequently used information was on fat, but information on sodium is widely neglected. Secondly, we found that ignorance and the attitude that labels are not useful were the main factors explaining the low use rate of all kinds of nutritional information 
in China. Chinese consumers ignore food labels, especially Nutrition Facts Panels because the content is complex and the labels are placed unobtrusively on the back of the packet. The poor location in the nutrition facts decreases its relevance and interest for consultation by consumers, and that frontal nutrition labeling could contribute to improving visibility. Besides, subjective understanding helps use the Nutrition Facts Panels, whereas objective understanding plays a significant role in the use of energy and protein information. Thus, the education program should be launched to improve both consumers' understanding ability and their subjective confidence in utilizing nutrition labels.

Our findings will have several implications in the design of nutrition labels especially for food processors targeting the Chinese market. First, food processors should use more simplified and striking tag formats, including Nutrition Claims and other front-of-pack labels, to improve consumer welfare and advertise product features. Brighter colors or larger fonts should also be considered by the food processors to highlight nutritional information statements. Second, sodium information can be emphasized when designing labels. 'Salt' may be used as a replacement of 'sodium' or 'Na' in the present nutrition labels. Because in this survey Chinese consumers reported that they are unfamiliar with the technical term 'Na' or 'sodium'. The order of display of nutrients in Nutrition Facts Panels should also be adjusted, such as placing sodium information first, so that it draws the attention of Chinese consumers.

These findings can also be used as a guide for food processors in the design of food marketing programs to advertise and promote directing at different consumer groups. In the first place, simplified front-of-pack labels should be emphasized especially for elderly people. Detailed back-of-pack labels can be applied when targeting people with a larger household size or higher education. Second, food advertising campaigns designed for different age and gender groups can be more effective. For example, stress on fat information targeting younger females but protein information targeting younger males. Finally, agribusiness firms could also considerably benefit from investing in nutrition campaigns in terms of their public image as they will contribute to more widespread adoption of healthy food selection.

\section{Supplementary material}

Supplementary material can be found online at https://doi.org/10.22434/IFAMR2020.0162

Table S1. Marginal effects for the use of energy and protein information.

Table S2. Marginal effects for the use of fat and carbohydrate information.

Table S3. Marginal effects for the use of sodium information.

\section{Acknowledgements}

The authors would like to thank research assistants Yue Jin, Changhua Qian, Haiyue Guo, Rao Yuan, Zhanyi Shi, Yu Jiang for their helpful support in data collection. We also gratefully acknowledge the funding support from the National Natural Science Foundation of China (grant number 72003175), the Major Program of the Key Research Institute of Chinese Ministry of Education (grant number No. 15JJD790032), and the Scientific Research Project of Zhejiang Education Department (grant number Y202043173). The authors have declared that no conflict of interest exists.

\section{References}

Akerlof, G.A. 1970. The market for 'lemons': quality uncertainty and the market mechanism. The Quarterly Journal of Economics 84(3): 488-500.

Aschemann-Witzel, J., K.G. Grunert, H.C.M. Van Trijp, S. Bialkova, M.M. Raats, C. Hodgkins, G. WasowiczKirylo and J. Koenigstorfer. 2013. Effects of nutrition label format and product assortment on the healthfulness of food choice. Appetite 71: 63-74. 
Barreiro-Hurlé, J., A. Gracia and T. De-Magistris. 2010. Does nutrition information on food products lead to healthier food choices. Food Policy 35: 221-229.

Blitstein, J.L. and W.D. Evans. 2006. Use of nutrition facts panels among adults who make household food purchasing decisions. Journal of Nutrition Education and Behavior 38(6): 360-364.

Bonanno, A. and S.J. Goetz. 2012. Food store density, nutrition education, eating habits and obesity. The International Food and Agribusiness Management Review 15(4): 1-26.

Boztuğ, Y., H.J. Juhl, H.J. Elshiewy and M.B. Jensen. 2015. Consumer response to monochrome guideline daily amount nutrition labels. Food Policy 53: 1-8.

Burton, S., J.A. Garretson and J.A. Velliquette. 1999. Implications of accurate usage of nutrition facts panel information for food product evaluations and purchase intentions. Journal of the Academy of Marketing Science 27(4): 470-480.

China's National Health and Family Planning Commission. 2015. Chinese residents nutrition and chronic disease status report. People's Medical Publishing House, Beijing, China.

Davidson, R. and J.G. MacKinnon. 1993. Estimation and inference in econometrics. Oxford University Press, Oxford, UK.

Drichoutis, A.C. 2005. Nutrition knowledge and consumer use of nutritional food labels. European Review of Agriculture Economics 32(1): 93-118.

Drichoutis, A.C., P. Lazaridis and R.M. Nayga. 2009. Would consumers value food-away-from-home products with nutritional labels? Agribusiness 25(4): 550-575.

Drichoutis, A.C., P. Lazaridis, R.M. Nayga, Jr., M. Kapsokefalou and G. Chryssochoidis. 2008. A theoretical and empirical investigation of nutritional label use. The European Journal of Health Economics 9(3): 293-304.

Grasso, S., F.J. Monahan, S.C. Hutchings and N.P. Brunton. 2017. The effect of health claim information disclosure on the sensory characteristics of plant sterol-enriched turkey as assessed using the checkall-that-apply (CATA) methodology. Food Quality and Preference 57: 69-78.

Grunert, K.G. and J.M. Wills. 2007. A review of European research on consumer response to nutrition information on food labels. Journal of Public Health 15(5): 385-399.

Hausman, J.A. 1978. Specification tests in econometrics. Econometrica 46(6): 1251-1271.

Houghton, J.R., G. Rowe, L.J. Frewer, E. Van Kleef, G. Chryssochoidis, O. Kehagia, S. Korzen-Bohr, J. Lassen, U. Pfenning and A. Strada. 2008. The quality of food risk management in Europe: perspectives and priorities. Food Policy 33(1): 13-26.

Khandpur, N., D.J. Graham and C.A. Roberto. 2017. Simplifying mental math: changing how added sugars are displayed on the nutrition facts label can improve consumer understanding. Appetite 114: 38-46.

Kim, H.S., C. Oh and C. No. 2016. Can nutrition label recognition or usage affect nutrition intake according to age? Nutrition 32(1): 56-60.

Kim, S.Y., R.M. Nayga and O. Capps. 2000. The effect of food label use on nutrient intakes: an endogenous switching regression analysis. Journal of Agricultural and Resource Economics 25(1): 215-231.

Kline, P. 1993. The handbook of psychological testing. Routledge, London, UK.

Lin, B., J.F. Guthrie, I. Rahkovsky, C. Lin and J. Lee. 2014. Simulating the potential effects of a shelf-tag nutrition information program and pricing on diet quality associated with ready-to-eat cereals. The International Food and Agribusiness Management Review 17: 7-24.

Lin, B.H. and S.T. Yen. 2008. Consumer knowledge, food label use and grain consumption in the US. Applied Economics 40(4): 437-448.

Liu, R., C.A. Hoefkens and W. Verbeke. 2015. Chinese consumers' understanding and use of a food nutrition label and their determinants. Food Quality and Preference 41: 103-111.

Long, J.S. 1997. Regression models for categorical and limited dependent variables. Sage Publications, Thousand Oaks, CA, USA.

Louzada, M.L.C., L.G. Baraldi, E.M. Steele, A.P.B. Martins, D.S. Canella, J.C. Moubarac, R.B. Levy, G. Cannon, A. Afshin, F. Imamura, D. Mozaffarian and C.A. Monteiro. 2015. Consumption of ultraprocessed foods and obesity in Brazilian adolescents and adults. Preventive Medicine 81: 9-15. 
Machado, P.P., R.M. Claro, D.S. Canella, F.M. Sarti and F.M. Levy. 2017. Price and convenience: the influence of supermarkets on consumption of ultra-processed foods and beverages in Brazil. Appetite 116: 381-388.

Miller, L.M. and D.L. Cassady. 2012. Making healthy food choices using nutrition facts panels. The roles of knowledge, motivation, dietary modifications goals, and age. Appetite 59(1): 129-139.

Moore, S.G., J.K. Donnelly, J.K. Jones and J.E. Cade. 2018. Effect of educational interventions on understanding and use of nutrition labels: a systematic review. Nutrients 10: 1432-1445.

Moubarac, J.C., A.P. Martins, R.M. Claro, R.B. Levy, G. Cannon and C.A. Monteiro. 2013. Consumption of ultra-processed foods and likely impact on human health. Evidence from Canada. Public Health Nutrition 16(12): 2240-2248.

National Bureau of Statistics of China. 2014. China statistical yearbook. China Statistics Press, Beijing, China. National Bureau of Statistics of China. 2015. China statistical yearbook. China Statistics Press, Beijing, China.

NCD Risk Factor Collaboration. 2016. Trends in adult body-mass index in 200 countries from 1975 to 2014: a pooled analysis of 1698 population-based measurement studies with 19.2 million participants. The Lancet 387(10026): 1377-1396.

Peschel, A.O., J.L. Orquin and S. Mueller Loose. 2019. Increasing consumers' attention capture and food choice through bottom-up effects. Appetite 132: 1-7.

Peterson, B. and F.E. Harrell. 1990. Partial proportional odds models for ordinal response variables. Applied Statistics 39(2): 205-217.

Popkin, B.M. 2014. Nutrition, agriculture and the global food system in low and middle income countries. Food Policy 47: 91-96.

Rimal, A., S.M. Fletcher and K.H. Mcwatters. 2000. Nutrition considerations in food selection. The International Food and Agribusiness Management Review 3(1): 55-70.

Roe, B.E., M.F. Teisl and C.R. Deans. 2014. The economics of voluntary versus mandatory labels. The Annual Review of Resource Economics 6: 407-427.

Shimokawa, S. 2016. Why can calorie posting be apparently ineffective? The roles of two conflicting learning effects. Food Policy 64: 107-120.

Sun, P.-C., J.A. Huang and F.-Y. Chu. 2015. Factors instead of demographic characteristics related to nutrition label use. British Food Journal 117(12): 3024-3038.

Unnevehr, L.J. and E. Jagmanaite. 2008. Getting rid of trans fats in the US diet: policies, incentives and progress. Food Policy 33(6): 497-503.

Verbeke, W. 2006. Functional foods: consumer willingness to compromise on taste for health? Food Quality and Preference 17: 126-131.

Wang, E.Y., H. Wei and J.A. Caswell. 2016. The impact of mandatory trans fat labeling on product mix and consumer choice: a longitudinal analysis of the U.S. market for margarine and spreads. Food Policy 64: 63-81.

Wang, S., P. Marquez and J. Langenbrunner. 2011. Toward a healthy and harmonious life in China: stemming the rising tide of non-communicable diseases. The World Bank, Washington, DC, USA.

Williams, R. 2006. Generalized ordered logit/partial proportional odds models for ordinal dependent variables. The Stata Journal 6(1): 58-82.

Zhou, Y., S. Du, C. Su, B. Zhang, H. Wang and B.M. Popkin. 2015. The food retail revolution in China and its association with diet and health. Food Policy 55: 92-100. 